\title{
Descripción de la primera curva epidémica por la Covid-19 en Costa Rica
}

\section{ARTÍCULO ORIGINAL BREVE}

\author{
Description of the first epidemic curve due to Covid-19 in Costa Rica
}

Robinson Rodríguez-Herrera $\square^{1,2}$, Enrique Víctor-Mora ${ }^{1,2}$, Rocío Valverde-Gallegos².

\section{Filiación:}

${ }^{1}$ Programa Institucional de Calidad y Seguridad del Paciente-PICSP, Caja Costarricense del Seguro Social, San José, Costa Rica.

${ }^{2}$ Universidad Santa Paula, San José, Costa Rica.

Correspondencia: $\square$ Robinson Rodríguez-Herrera. Correo electrónico: drrobinsongerenciasalud@gmail.com

Financiamiento:

Ninguno

Conflictos de Interés:

Ninguno

Forma de citar: Rodríguez-Herrera $R$, Victor-Mora E, Valverde-Gallegos $R$. Descripción de la primera curva epidémica por la Covid-19 en Costa Rica. Rev Ter. 2021;15(1): 96-106

Abreviaturas: CCSS: Caja costarricense del Seguro Social; SARS-CoV-2: coronavirus del síndrome respiratorio agudo grave tipo 2; $A R N$ : ácido ribonucleico.

Fecha de recepción: 26 de diciembre del 2020.

Fecha de aceptación: 22 enero del 2021.

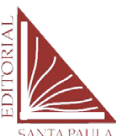

\section{Resumen}

Introducción: el objetivo del presente manuscrito fue describir las principales características epidemiológicas de la denominada primera curva epidémica causada por la enfermedad Covid-19 en Costa Rica entre 06 de marzo y hasta el 24 de abril del 2020. Metodología: se realizó un estudio observacional descriptivo de los casos de pacientes con enfermedad por la COVID-19 en Costa Rica durante las semanas epidemiológicas 10 a la 17. Los datos que se utilizan en este trabajo fueron tomados y resumidos de los reportes, informes diarios y actualizaciones que se publican en los sitios de dominio público de la Caja Costarricense del Seguro Social y el Ministerio de Salud de Costa Rica, instituciones que tienen el mandato constitucional y legal de la atención y la vigilancia de la salud, respectivamente, en todo el territorio nacional. Estos datos se consolidaron y procesaron en una hoja de cálculo de Excel ${ }^{\circledR}$. Resultados: al final de esos 50 días de la primera curva, hubo un total de 687 pacientes confirmados, entre los 1 y 87 años, $52 \%$ masculinos $(n=359)$, afectando al $0,013 \%$ de los habitantes censados del país (n: 5111238 ) con una letalidad del $0,87 \%$. El promedio de casos nuevos diarios fue de 13,8 , la mediana de 16 y la moda 19. $90 \%$ de los casos fueron nacionales $(n=620)$ y el 10\% ( $n=67)$ eran extranjeros, incluyendo residentes formales. La distribución según edad fue de un $91 \%$ de pacientes adultos $(n=618)$, un $5,1 \%$ de adultos mayores $(n=35)$ y un $4,9 \%$ de menores de edad $(n=34)$. Durante la primera curva epidémica, se logró realizar el seguimiento de los casos y establecer los conglomerados, lo cual facilitó las labores de atención por parte del personal de salud. Conclusión: a diferencia de otros países, la primera curva epidémica en Costa Rica representó un periodo particular de bajo estrés (pocos casos y baja letalidad de estos) para el sistema nacional de salud, que pudo ser aprovechado para fortalecer las intervenciones en comunidades de riesgo potencial, tales como asentamientos humanos ubicados en plantaciones fronterizas, tugurios y cuarterías, y también para reforzar la educación para la salud en la población, así como dotar al sistema de salud de mayores recursos para atender la segunda ola epidémica.

Palabras clave: Covid-19, pandemia, epidemiología, Costa Rica. 


\section{Abstract}

Introduction: the objective of this manuscript was to describe the main epidemiological characteristics of the socalled first epidemic curve caused by the Covid-19 disease in Costa Rica between March 6 and April 24, 2020. Results: at the end of those 50 days of the first curve, there were a total of 687 confirmed patients, between the ages of 1 and $87,52 \%$ male $(n=359)$, affecting $0.013 \%$ of the country's registered inhabitants ( $n: 5111238)$ with a lethality of $0.87 \%$. The average number of new cases per day was 13.8 , the median was 16 , and the mode was 19. $90 \%$ of the cases were nationals $(n=620)$ and $10 \%(n=67)$ were foreigners, including formal residents. The age distribution was $91 \%$ of adult patients $(n=618), 5.1 \%$ of older adults $(n=35)$ and $4.9 \%$ of minors $(n=34)$. During the first epidemic curve, it was possible to monitor the cases and establish the clusters, which facilitated the work of care by health personnel. Conclusion: unlike other countries, the first epidemic curve in Costa Rica represented a particular period of low stress (few cases and low lethality of these) for the national health system, which could be used to strengthen interventions in at-risk communities potential, such as human settlements located in border plantations, slums and barracks, and also to reinforce health education in the population, as well as provide the health system with more resources to attend the second epidemic wave.

Keywords: Covid-19, pandemic, epidemiology, Costa Rica.

\section{Introducción}

La actual pandemia causada por el virus SARS-CoV-2 se identificó formalmente por primera vez en la ciudad de Wuhan, ubicada en China, en el mes de diciembre del año 20191,2. A partir de ese momento se documenta la diseminación de la enfermedad por diversos países del mundo, aunque la Organización Mundial de la Salud (OMS) declaró que había alcanzado el nivel de pandemia hasta el 11 de marzo del año $2020^{3,4}$.

El virus SARS-CoV-2 (coronavirus del síndrome respiratorio agudo grave tipo 2) pertenece a la familia de los coronavirus que son virus ARN. Este virus está compuesto por una sola cadena de ARN y con una longitud de alrededor de 30000 bases y las glicoproteínas de su cubierta son las que le dan el aspecto del cual deriva el nombre de coronavirus ${ }^{5,6}$. A diferencia de otros patógenos que han causado pandemias catastróficas en el transcurso de la historia de las civilizaciones, como por ejemplo el virus de la gripe H1N1 en 1918, este virus no presenta hasta el momento una periodicidad estacional y, por tanto, a partir de su primer contagio documentado en la historia no ha requerido de reservorios animales por medio de los cuales mutar y también transmitirse a otras especies, entre las cuales se encuentra el ser humano, aunque no se puede descartar que en un futuro inmediato encuentre reservorios a partir de los cuales llegue a diseminarse ${ }^{6,7}$. El reservorio original se estableció que eran los murciélagos Rhinolophus affinis, conocidos como murciélago de herradura ${ }^{8,9}$, pero el riesgo es latente pues es conocido que los coronavirus en general afectan a una amplia variedad de especies con las cuales el ser humano tiene contacto en sus ambientes domésticos, agroindustriales y naturales. Un ejemplo reciente fue el caso ocurrido en algunas granjas de visones en Europa ${ }^{10}$.

La transmisión de este patógeno se realiza fundamentalmente por medio de las secreciones que viajan dispersas en microgotas producto de la función respiratoria y el habla normal, y no 
exclusivamente por medio de la tos o el estornudo ${ }^{11}$. También se ha descrito que sobrevive en superficies por tiempos generalmente cortos en el transcurso de los cuales conserva su potencial infeccioso si es puesto luego en contacto con membranas mucosas, para las cuales presenta afinidad ${ }^{11,12}$. Hasta la fecha no se ha demostrado que pueda ser transmitido por vectores a diferencia de otras enfermedades como el dengue, el zika y el chikungunya ${ }^{12,13}$.

Entre los síntomas reportados como más frecuentes están: fiebre, tos, fatiga, cefalea, diarrea, hemoptisis y disnea ${ }^{14}$.

La curva que representa a una epidemia generalmente va a caracterizarse por una distribución que es asimétrica, en la que se observa una fase ascendente relacionada con la forma como el agente infeccioso se propaga en una población susceptible determinada. Esta curva asciende hasta llegar a un tope y luego presenta una fase descendente que está condicionada también por las características de la propagación y la susceptibilidad de población en la cual se manifiesta ${ }^{15}$. Tanto en una epidemia como en una pandemia, la transmisión comunitaria representa un serio problema para los sistemas sanitarios debido a la mayor exigencia de consultas, tratamiento e internamientos que ponen en serio riesgo de colapso a los establecimientos y personal de atención de la salud, además del aumento de los costos que es un factor crítico especialmente para países en vías de desarrollo. Ante un aumento significativo de los casos, conseguir la cantidad suficiente de insumos y de equipos indispensables que cumplan los requisitos de calidad y seguridad, puede resultar difícil o incluso imposible. Por tanto, los periodos en los cuales la transmisión y la incidencia de los casos son bajos, representan oportunidades muy valiosas para implementar estrategias preventivas en la población y para reforzar las fortalezas del sistema de salud.

\begin{tabular}{c}
\hline Metodología \\
\hline Se realizó un estudio transversal
\end{tabular}
retrospectivo descriptivo de los casos de pacientes con enfermedad por la COVID-19 en Costa Rica durante las semanas epidemiológicas 10 a la 17 (entre 06 de marzo y hasta el 24 de abril del 2020). Los datos que se utilizaron en este trabajo fueron tomados de los reportes, informes diarios y actualizaciones que se publicaron en los sitios de dominio público de la Caja Costarricense del Seguro Social $(\mathrm{CCSS})^{16}$ y el Ministerio de Salud de Costa Rica (MINSA) ${ }^{17}$, instituciones que tienen el mandato constitucional y legal de la atención y la vigilancia de la salud, respectivamente, en todo el territorio nacional. Estos datos se consolidaron y procesaron en una hoja de cálculo de Excel®.

Consideraciones éticas: el presente estudio fue realizado a partir de datos filtrados y publicados por el sistema nacional de atención de la salud de Costa Rica, por lo que trabajar con esos datos procurando consolidarlos para generar información de utilidad para el análisis no expone la confidencialidad de ninguno de los pacientes y sus familias. Por tanto, no existe conflicto con los principios bioéticos que rigen la investigación en salud pública.

\section{Resultados}

El primer caso diagnosticado y confirmado por laboratorio de COVID-19 en Costa Rica se anunció el día 06 de marzo del 2020 (fecha que se tomará como el día 1 de la llegada de la pandemia al país, coincidiendo con la semana epidemiológica 10), y que corresponde a una paciente que había ingresado con propósitos de turismo el 01 de marzo, 
viajando en un vuelo comercial, y sin manifestar ningún síntoma hasta unos días después de su arribo $^{18}$. En el mundo, el primer caso diagnosticado formalmente se documentó el día 17 de noviembre de 2019, por tanto, a nuestro país el contagio llegó 111 días después. En México el primer caso se documentó el día 8 de enero de $2020^{19}$.

A partir del primer caso, la cantidad de casos nuevos diarios se mantuvo baja (4,2 casos diarios en promedio) hasta trece días después, cuando llega a 19 casos nuevos diarios e inicia un aumento sostenido de la incidencia con un máximo de 37 casos el día 09 de abril (día 35). A partir de ese momento sigue un descenso paulatino presentando su punto más bajo el día 24 de abril (día 50, coincidiendo con la semana epidemiológica 17), cuando se reporta solamente un caso nuevo. Esto se ilustra en la figura 01. La tasa R del COVID-19 para el 24 de abril era de $0,793{ }^{20}$.

Al final de esos 50 días de la primera curva, el total de pacientes confirmados fue de 687, dentro de un rango de edad de 01 a 87 años $^{21}$, que corresponden al 0,013\% de los habitantes censados del país que son $5111238^{22}$. El promedio de casos nuevos diarios fue de 13,8 , el máximo fue de 37 , el mínimo de 01, la mediana de 16 y la moda 19. $52 \%$ de casos fueron masculinos ( $n=359$ ), $90 \%$ de los casos fueron nacionales ( $n=620$ ), mientras que $10 \%(n=67)$ eran extranjeros, incluyendo residentes formales. Esto es importante de mencionar pues durante este tipo de situaciones son frecuentes las actitudes xenofóbicas en algunas personas, las cuales no se justifican pues la mayor parte de los casos ocurrió en pacientes nacionales.

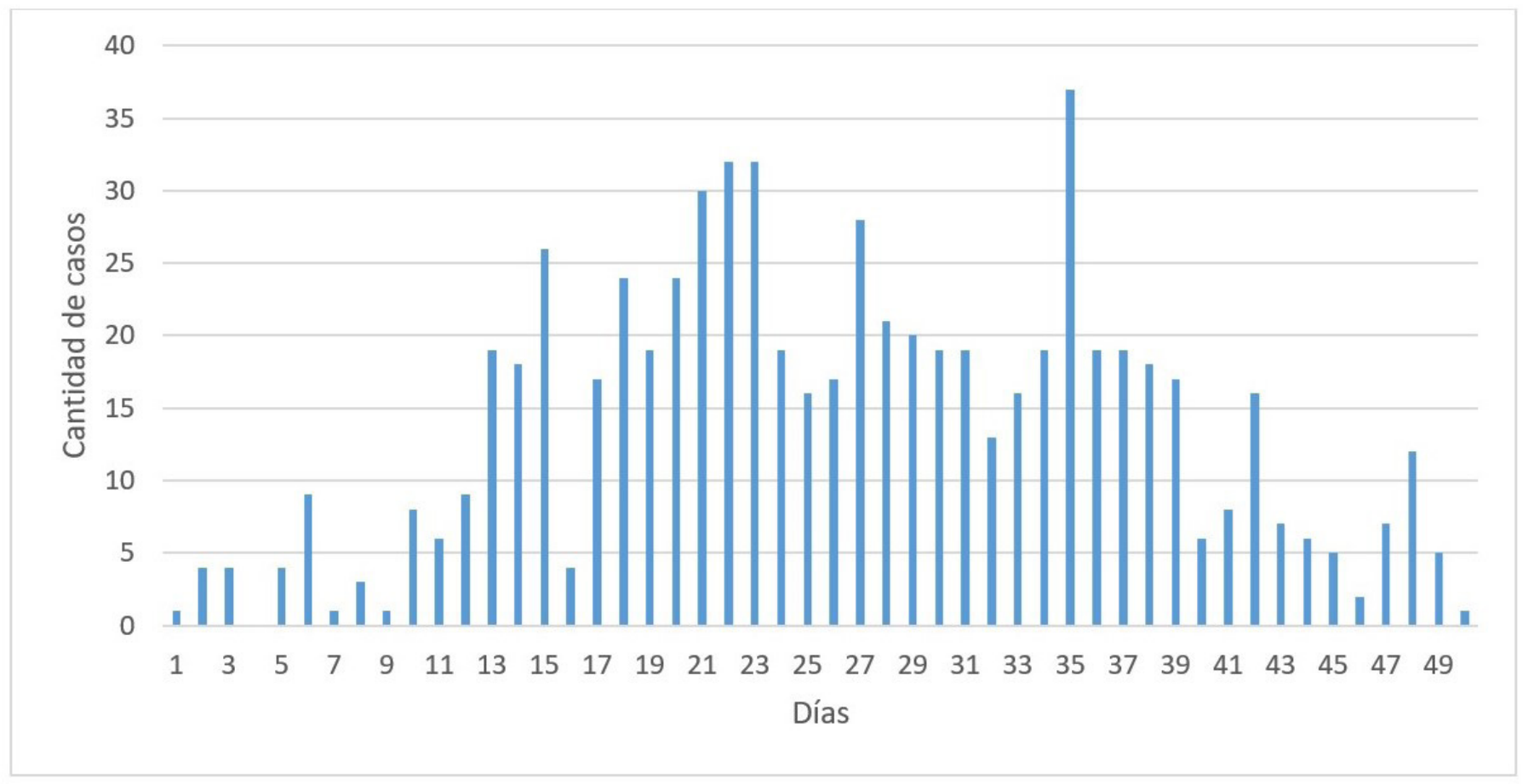

Figura 1. Distribución de casos durante la primera curva epidémica causada por la enfermedad Covid-19 en Costa Rica, marzo-abril 2020.

Fuente: elaborado por los autores a partir de datos publicados por el MINSA y la CCSS en el 2020. 
La distribución según edad fue de un $91 \%$ de pacientes adultos ( $n=618$ ), un $5,1 \%$ de adultos mayores $(n=35)$ y un $4,9 \%$ de menores de edad $(n=$ 34).

Al final de esta primera curva se habían procesado 12324 muestras en los laboratorios, y del total de casos investigados en ese momento se descartaron 8133. No se realizaron pruebas masivas a diferencia de otros países. Oficialmente se contabilizaron 216 pacientes recuperados, cuyo rango de edad estaba entre 01 y 86 años, sin que se presentaran diferencias significativas según sexo: $52 \%$ de masculinos $(n=113)$ y un $48 \%$ de pacientes femeninas $(n=103)$. La distribución según grupos de edad de esos pacientes recuperados fue de un $88 \%$ de adultos $(n=190)$, un $6 \%$ de adultos mayores $(n=13)$ y un $6 \%$ de menores de edad $(n=13)$, lo cual es concordante con los casos diagnosticados.

Respecto de la distribución geográfica en ese momento, ya se habían confirmado casos en las 7 provincias del país, con un predominio en las provincias geográficamente relacionadas con el Valle Central del país, que son las de mayor densidad poblacional. La distribución de casos según provincia se detalla en la figura 2.

Respecto de los casos por cantón, se confirmaron casos en $72 \%$ (59/82) de los cantones que conforman el país. En el $80 \%$ de esos 59 cantones ya se reportaban pacientes recuperados al final de la curva epidémica descrita. Los cantones con mayor cantidad de casos en ese momento eran: San José con 95, Alajuela con 75, Desamparados con 47, y Santa Ana con $46^{23}$.

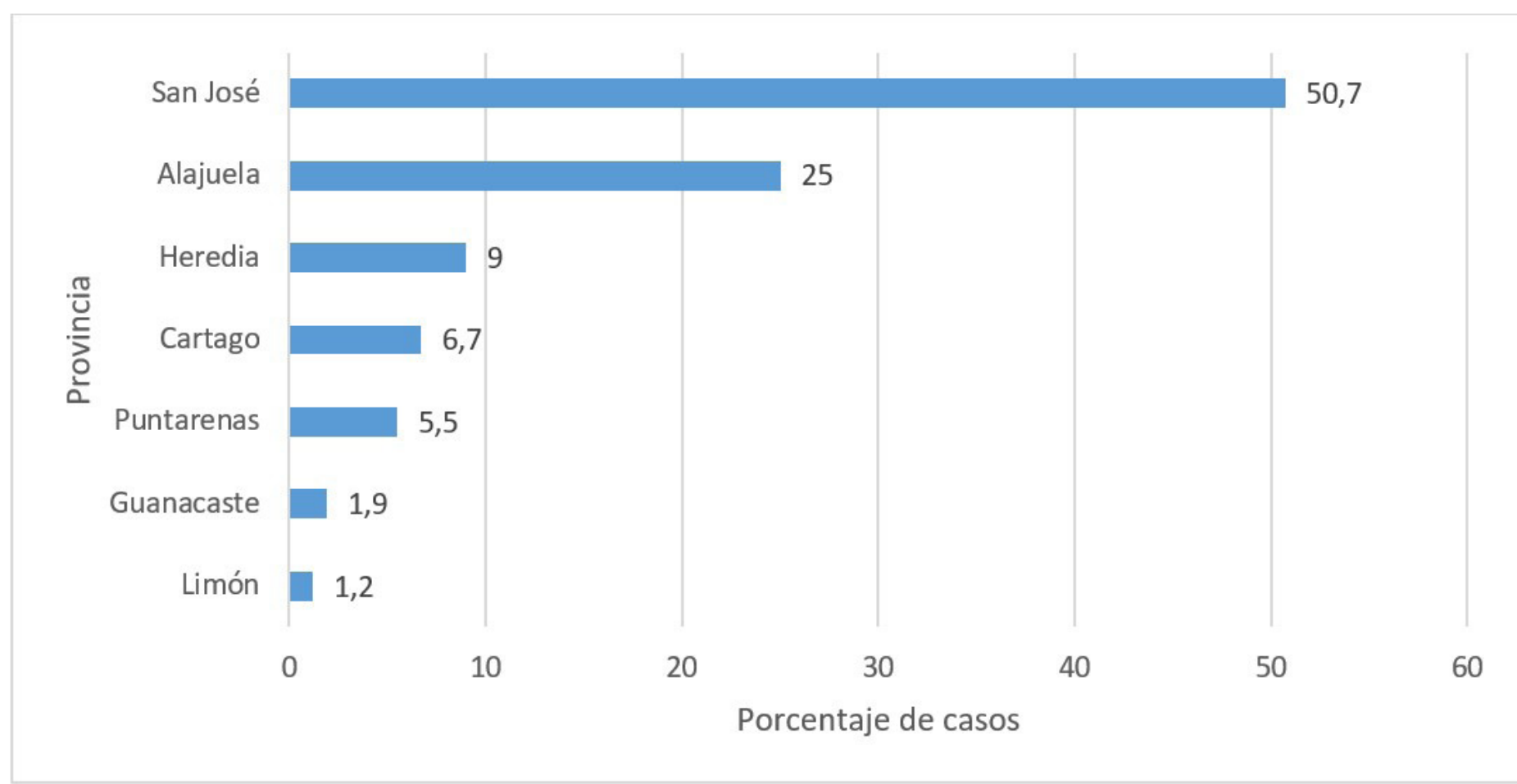

Figura 2. Distribución porcentual de casos por provincia durante la primera curva epidémica causada por la enfermedad Covid-19 en Costa Rica. Marzo-abril, 2020.

Fuente: elaborado por los autores a partir de datos publicados por el MINSA y la CCSS en el 2020. 


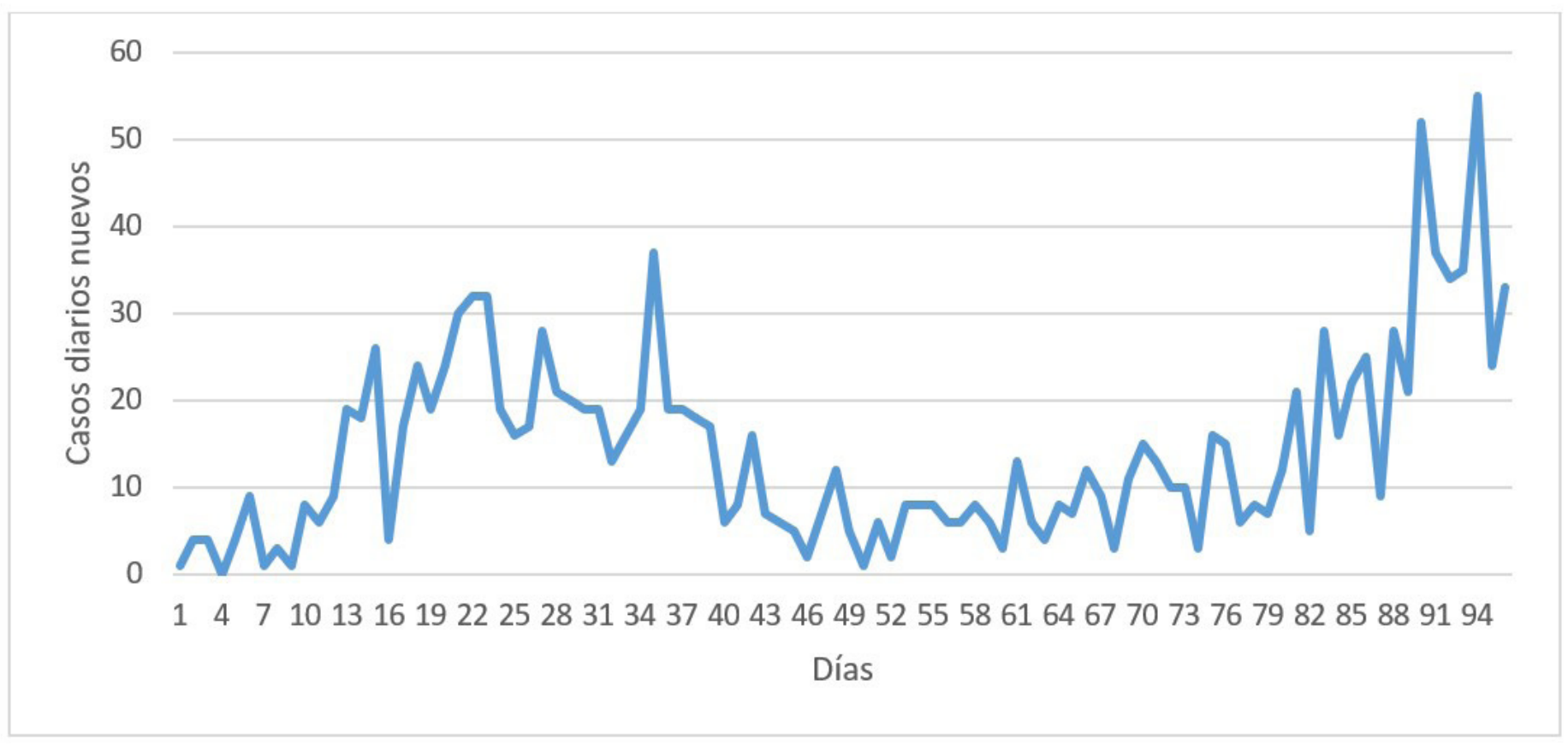

Figura 3. Distribución de casos diarios nuevos durante la enfermedad Covid-19 en Costa Rica en el año 2020 hasta el día 96 desde el primer caso.

Fuente: elaborado por los autores a partir de datos publicados por el MINSA y la CCSS en el 2020.

Del total de los 687 casos confirmados oficialmente por las autoridades de salud en ese período fallecieron 6 personas, lo que corresponde a una tasa de letalidad de $0,87 \%$. La totalidad de los decesos correspondieron a pacientes masculinos, ubicados en un rango de edad de 45 a 87 años y un promedio de 75,7 años. Para el 24 de abril 12 personas estaban hospitalizadas, 7 de las cuales (58\%) se encontraban en las unidades de cuidados intensivos de hospitales de alta complejidad ubicados en el Valle Central, ocupando el $29 \%$ de la capacidad de estas. Para ese entonces se había iniciado el aumento de camas de Unidades de Cuidado Intensivo, que en febrero eran 24 , distribuidas de la siguiente forma: Hospital México: 12, Hospital San Juan de Dios: 7 y el Hospital Calderón Guardia con 5. Posteriormente se han habilitado para el mes de septiembre 211 unidades $\mathrm{UCl}$ y se esperaba llegar a disponer de más de $287^{24,25}$.

De los pacientes que fallecieron, 5 de los 6
(83\%) presentaban al menos un factor de riesgo, entre los cuales se documentaron hipertensión arterial, diabetes, tabaquismo y tener más de 65 años. Un paciente fallecido, masculino de 45 años, no se le documentó ningún factor de riesgo.

Otra particularidad de esta primera curva es la cantidad de linajes virales que se lograron secuenciar y dar seguimiento, por parte de investigadores del Instituto Costarricense de Investigación y Enseñanza en Nutrición y Salud (Inciensa) y de la Universidad de Costa Rica $(\mathrm{UCR})^{26}$, quienes durante marzo a julio del 2020, realizaron una investigación en la que de un total de 70 genomas virales presentes en el país lograron la secuenciación de 06 genomas completos del virus SARS-CoV-2 y encontraron que en ese momento predominaron en la población afectada tres linajes virales: B.1 (49\%), B.1.5 (21\%) y B.1.1. (19\%). Basados en estos datos, una de las conclusiones a las que estos investigadores llegaron es que los 
linajes se relacionaban con pacientes que estaban llegando al país procedentes de varias regiones del extranjero, principalmente de Norteamérica, América del Sur y Europa.

A partir de ese día 50 desde el inicio de la pandemia en el país, la cantidad de casos nuevos presenta un descenso y mantiene una estabilidad relativa, con un promedio de 15,1 casos diarios, y así se mantiene hasta el mes de junio (09 de junio, día 96, correspondiente a la semana epidemiológica 24), a partir de este momento es que consideramos que inicia la fase ascendente de la segunda curva, caracterizada por una cantidad de casos diarios mucho mayor, lo que advierte de un cambio en la velocidad de los contagios, característica que es sugestiva del inicio de la transmisión comunitaria, circunstancia en la que no se pueden identificar ya los conglomerados para darles atención integral y procurar detener su diseminación. El aumento ocurre a pesar de que las medidas generales de restricción sanitaria todavía se mantenían desde la declaración de la emergencia nacional, contenida en el Decreto Ejecutivo 42227-MP-S del 16 de marzo del 2020 que restringía las horas, días y zonas de tránsito vehicular y también el cierre de establecimientos públicos y privados.

La figura 3 ilustra cómo del día 34 al día 50 hubo una caída drástica de número de casos, que marca el término de la primera ola, y días después se aprecia el ascenso de los casos que representa el inicio de la segunda ola epidémica en el país.

\section{Discusión}

Esta primera curva de la enfermedad viral pandémica ocurrió en el país en una época caracterizada por la ausencia de controles sanitarios particulares a los viajeros a pesar del conocimiento de la pandemia y su abordaje integral en otros países por parte de las autoridades de salud y agencias gubernamentales en general. Debido a la poca cantidad de casos, es que se logró darle atención con calidad y realizar un buen seguimiento a los conglomerados que iban apareciendo. Sin embargo, durante esa primera curva no se realizaron testeos masivos $^{27}$ y tampoco se hizo obligatorio el uso de mascarillas en sitios públicos.

Como un ejemplo de la particularidad de esta primera curva en Costa Rica, si nos comparamos con otro país de la región, concretamente en el caso de Panamá, resulta que al 1 de abril el Ministerio de Salud de este país vecino había reportado unos 1.200 enfermos y 30 muertes como consecuencia del covid-19 ${ }^{28}$, casos en los cuales las autoridades de salud no podían identificar los conglomerados. En tanto en Costa Rica para ese mismo día se reportaban 375 casos acumulados en total y solamente 2 fallecimientos, todos ubicados en conglomerados a los cuales se les daba seguimiento. Esto ilustra sobre la particularidad y relativa benevolencia con la que se presentó la enfermedad inicialmente en Costa Rica. La letalidad general en México hasta mayo del 2020 fue de $10.02 \%^{29}$, en tanto en Costa Rica al final de esa primera curva, la letalidad general era de $0,87 \%$.

Hasta el día 18 de marzo (día 44), el gobierno ordenó el cierre de las fronteras marítimas, terrestres y aéreas del país, medida que no se elimina por completo sino hasta el 01 de noviembre del 2020. Posteriormente, pretendiendo disminuir los contagios el gobierno decretó una restricción diferenciada en la Gran Área Metropolitana aplicando restricciones de circulación y comercio inicialmente del 11 al 19 de julio. Es importante mencionar que no es sino hasta el día 20 de julio, semana epidemiológica 30 , cuando se declara la obligatoriedad del uso de mascarillas en lugares públicos. 
Esta primera curva epidémica en Costa Rica ocurrió en los meses de marzo y abril del 2020, y se caracterizó por ser limitada tanto en tiempo como en cantidad de pacientes. También en su amplitud, impacto para el sistema nacional de salud y respecto de la mortalidad que causó. La mayor proporción de afectados correspondió a pacientes adultos masculinos, y la mayor cantidad de pacientes se ubicó en la capital y en cantones del valle central. La mayoría de los pacientes fallecidos presentaban al menos un factor de riesgo. Tres linajes virales: B.1 (49\%), B.1.5 (21\%) y B.1.1. (19\%) predominaron en ese momento. En atención al número de internamientos y atención de pacientes en general, se concluye que la cantidad de contagios y la evolución clínica de la mayoría de estos no llegó a poner en compromiso la capacidad hospitalaria de la Seguridad Social.

A la fecha, gran cantidad de artículos se han publicado en el mundo sobre diversas particularidades de la pandemia, a nivel global y en los países o regiones. Hay consenso en los autores respecto de que las medidas de aislamiento y la aplicación de las pruebas masivas son medidas que han demostrado ser efectivas en otros países para el control de la transmisión de esta enfermedad ${ }^{30,31}$.

La primera curva epidémica por COVID-19 en Costa Rica, representó un periodo particular de bajo estrés para el sistema nacional de salud que podía ser aprovechado para fortalecer las intervenciones en comunidades de riesgo potencial, tales como asentamientos humanos ubicados en plantaciones fronterizas, tugurios y cuarterías, y también para reforzar la educación para la salud en la población.

\section{Referencias bibliográficas}

1. Organización Mundial de la Salud. Coronavirus disease 2019 (COVID-19) Situ- ation Report -94 [Internet]. Ginebra: OMS; 2020. Disponible en: https://www.who.int/ docs/default-source/coronaviruse/situation-reports/20200423-sitrep-94-covid-19. pdf

2. Yin C. Genotyping coronavirus SARS-CoV-2: methods and implications. Genomics. 2020;112(5):3588-3596. doi: 10.1016/j.ygeno.2020.04.016.

3. Cucinotta D, Vanelli M. WHO Declares COVID-19 a Pandemic. Acta Biomed. 2020 19;91(1):157-160. doi: 10.23750/abm. v91i1.9397

4. Adhamon T. Organización Mundial de la Salud [Internet]. Ginebra: OMS; 2020. WHO: Director-General's opening remarks at the media briefing on COVID19 -March 2020; [aprox. 5 pantallas]. Disponible en: https://www.who.int/director-general/speeches/detail/who-director-general-s-openingremarks-at-the-media-briefing-on-covid-1911-march-2020

5. Huang Y, Yang C, Xu XF, Xu W, Liu SW. Structural and functional properties of SARS-CoV-2 spike protein: potential antivirus drug development for COVID-19. Acta Pharmacol Sin. 2020;41(9):1141-1149. doi: 10.1038/s41401-020-0485-4.

6. Virological [Internet]. Reino Unido: Virological.org; 2020. Novel 2019 Coronavirus Genome. Disponible en: https://virological. org/t/novel-2019-coronavirus-genome/319

7. Du Toit A. Outbreak of a novel coronavirus. Nat Rev Microbiol. 2020;18(3):123. doi: 10.1038/s41579-020-0332-0 
8. Zhou $P$, Yang $X L$, Wang $X G$, Hu $B$, Zhang $L$, Zhang $W$, et al. A pneumonia outbreak associated with a new coronavirus of probable bat origin. Nature. 2020;579(7798):270-273. doi: 10.1038/ s41586-020-2012-7

9. Lam TT, Jia N, Zhang YW, Shum MH, Jiang JF, Zhu HC, et al. Identifying SARSCoV-2-related coronaviruses in Malayan pangolins. Nature. 2020;583(7815):282-285. doi: 10.1038/s41586-020-2169-0.

10. Munnink BBO, Sikkema RS, Nieuwenhuijse DF, Molenaar RJ, Munger E, Molenkamp $\mathrm{R}$, et al. Jumping back and forth: anthropozoonotic and zoonotic transmission of SARS-CoV-2 on mink farms. BioRxiv. 2020. doi: 10.1101/2020.09.01.277152v1

11. Chen J. Pathogenicity and transmissibility of 2019-nCoV-A quick overview and comparison with other emerging viruses. Microbes Infect. 2020;22(2):69-71. doi: 10.1016/j.micinf.2020.01.004

12. Organización Mundial de la Salud. Transmisión del SARS-CoV-2: repercusiones sobre las precauciones en materia de prevención de infecciones [Internet]. Ginebra: OMS; 2020. Disponible en: https://apps. who.int/iris/bitstream/handle/10665/333390/ WHO-2019-nCoV-Sci_Brief-Transmission_ modes-2020.3-spa.pdf

13. Organización Mundial de la Salud [Internet]. Ginebra: OMS; 2020. Vías de transmisión del virus de la COVID-19: repercusiones para las recomendaciones relativas a las precauciones en materia de pre- vención y control de las infecciones; [aprox. 8 pantallas]. Disponible en: https://www. who.int/es/news-room/commentaries/detail/ modes-of-transmission-of-virus-causing-covid-19-implications-for-ipc-precaution-recommendations

14. Adhikari SP, Meng S, Wu YJ, Mao YP, Ye RX, Wang QZ, et al. Epidemiology, causes, clinical manifestation and diagnosis, prevention and control of coronavirus disease (COVID-19) during the early outbreak period: a scoping review. Infect Dis Poverty. 2020;9(1):29. doi: 10.1186/s40249-02000646-x.

15. Organización Panamericana de la Salud. Módulo de Principios de Epidemiología para el Control de Enfermedades (MOPECE). Unidad 4: Vigilancia en Salud Pública [Internet]. 2a ed. Washington: OPS; 2011. Disponible en: https://www.paho. org/col/index.php?option=com_docman\&view=download\&category_slug=publicaciones-ops-oms-colombia \&alias=856- $\mathrm{mope}$ ce4\&ltemid $=688$

16. Caja Costarricense del Seguro Social [Internet]. San José: CCSS; 2020. COVID-19; [aprox. 5 pantallas]. Disponible en: https://www.ccss.sa.cr/web/coronavirus/index

17. Ministerio de Salud (CR) [Internet]. San José, CR: Ministerio de Salud; 2020. Situación Nacional Covid-19; [aprox. 6 pantallas]. Disponible en: https://www.ministeriodesalud.go.cr/index. php/centro-de-prensa/noticias/741-noticias-2020/1725-situacion-nacional-covid-19 
18. Caja Costarricense del Seguro Social [Internet]. San José: CCSS; 2020. Enfermedad Covid19 por el nuevo coronavirus; [aprox. 5 pantallas]. Disponible en: https://www.ccss.sa.cr/web/coronavirus/?gclid=EAlalQobChMIqai4xM_G7QIVxDizAB2DsgmHEAAYASAAEgl-xPD_BwE

19. Gobierno de México [Internet]. México: Gobierno de México; 2020. Coronavirus. Disponible en: https://coronavirus.gob.mx/

20. Universidad de Costa Rica, Centro Centroamericano de Población [Internet]. San José, CR: Centro Centroamericano de Población; 2020. Tasa R Covid-19; [aprox. 10 pantallas]. Disponible en: https://ccp.ucr. ac.cr/index.php/tasa-r-covid-19.html

21. Ministerio de Salud (CR) [Internet]. San José, CR: Ministerio de Salud; 2020. 687 casos confirmados por COVID-19; [aprox. 1 pantalla]. Disponible en: https://www.ministeriodesalud.go.cr/index.php/centro-de-prensa/noticias/741-noticias-2020/1630-687-casos-confirmados-por-covid-19

22. Instituto Nacional de Estadística y Censos (CR) [Internet]. San José, CR: INEC; 2020. Proyección de Población al 30 de junio de 2020; [aprox. 1 pantalla]. Disponible en: https://www.inec.cr/

23. Ministerio de Salud (CR) [Internet]. San José, CR: Ministerio de Salud; 2020. Situación Nacional COVID-19 : 24 Abril 2020; [aprox. 1 pantalla]. Disponible en: https:// geovision.uned.ac.cr/oges/historico_covid/ covid19_20200424.html
24. Bustamante Castillo X. Caja Costarricense del Seguro Social [Internet]. San José: CCSS; 2020. Mayor esfuerzo en acatar medidas sanitarias puede frenar velocidad de transmisión y mantener capacidad en servicios de salud; [aprox. 6 pantallas]. Disponible en: https://www.ccss.sa.cr/noticias/ servicios_noticia?mayor-esfuerzo-en-acatar-medidas-sanitarias-puede-frenar-velocidad-de-transmision-y-mantener-capacidad-en-servicios-de-salud

25. Rodríguez E. CCSS dispone de 912 respiradores y 211 camas $\mathrm{UCl}$ para atender pacientes con COVID-19 [Internet]. EI Observador. 01 de setiembre de 2020. Disponible en: https://observador.cr/noticia/ccss-dispone-de-912-respiradores-y-211-camas-ucipara-atender-pacientes-con-covid-19/

26. Aguilar Ramírez $P$, Enríquez Valencia Y, Quiroz Carrillo C, Valencia Ayala E, de León Delgado J, Pareja Cruz A. Pruebas diagnósticas para la COVID-19: la importancia del antes y el después. Horiz. Med [Internet]. 2020;20(2):e1231. doi: 10.24265/horizmed.2020.v20n2.14

27. Duarte Martínez F, Molina Mora JA, Cordero Laurent E, Godínez Rojas A, Calderón Osorno M, Corrales Aguilar E, et al. Análisis genómico de secuencias del virus SARS-CoV-2 de casos costarricenses [Internet]. San José, CR: Instituto Costarricense de Investigación y Enseñanza en Nutrición y Salud; 2020. Disponible en: https://www.inciensa.sa.cr/vigilancia_epidemiologica/informes_vigilancia/2020/Virologia/20200910_ SARS-CoV-2_wgs_informev2.pdf 
28. Datosmacro [Internet]. España: Datosmacro; 2020. Panamá - COVID-19 - Crisis del coronavirus; [aprox. 5 pantallas]. Disponible en: https://datosmacro.expansion. com/otros/coronavirus/panama

29. Padilla Santamaría F, Maya Franco L, Ferman Cano F. COVID-19 en México: Panorama Epidemiológico. Rev Cadena Cereb. 2020;4(1):31-42. doi: 10.5281/zenodo.3926806

30. Centers for Disease Control and Prevention (US) [Internet]. Atlanta: CDC; 2020. Interim Guidelines for Collecting, Handling, and Testing Clinical Specimens for COVID-19; [aprox. 9 pantallas]. Disponible en: https://www.cdc.gov/coronavirus/2019-ncov/lab/guidelines-clinical-specimens.html

31. Bchetnia M, Girard C, Duchaine C, Laprise C. The outbreak of the novel severe acute respiratory syndrome coronavirus 2 (SARS-CoV-2): A review of the current global status. J Infect Public Health. $2020 ; 13(11): 1601-1610$. doi: 10.1016/j. jiph.2020.07.011. 